\title{
VAC.0I - Carboxymethyl chitosan (CMCS) nanoparticles for mucosal vaccination against rabies: evaluation of the immune response following oral immunization studies in mice
}

Natalia Marchesan Bexiga ${ }^{1 *}$; Celso Pereira Caricati²; Marcos Vinicius Nucci Capone²; Adriano Mesquita Alencar ${ }^{1}$; Marco Antonio Stephano ${ }^{1}$.

1USP - Universidade de São Paulo;

2Instituto Butantan.

Introduction: Natural polysaccharides are widely being studied as biomaterials for drug delivery applications. Chitosan, the deacetylated form of chitin has a great potential to be used as a delivery system for antigens by mucosal surfaces. Chitosan nanoparticles have shown effective endocytotic uptake and low cytotoxicity using different cell models. However, limited colloidal stability, uncontrollable degradability, and the limited solubility in water or at $\mathrm{pH}$ higher than chitosan $\mathrm{pKa}$ ( $\mathrm{pH}$ 5.5-6.5) has prevented its full exploitation in the drug delivery field.

Objective: The purpose of this work was to assess the ability of inactivated rabies virus (RV) encapsulated in carboxymethyl chitosan (CMCS) nanoparticles to induce local and systemic immune responses following oral vaccination.

Methodology: Four groups of mice were used, a group treated with suspension of CMCS nanoparticles loaded with 10ug RVsAg (i.g.), a group treated with suspension of empty CMCS nanoparticles (i.g.), a group treated with saline solution of $0.9 \% \mathrm{NaCl}$ (i.g.), and a group treated with suspension of $10 \mathrm{ug}$ RVsAg intraperitoneally (i.p.). Mice were vaccinated on days 0, 14, and 21 , i.e. on weeks 1,3 , and 4 . The animals were sacrificed 7 days after the last immunization, and a collection of blood from the ophthalmic venous plexus and saliva was carried out for analysis. The RFFIT procedure was utilized to measure the level of rabies virus neutralizing antibody activity (RVNA) against the challenge virus standard 11 (CVS-11) strain of rabies virus in human serum samples. Statistical significance was assessed using one-way ANOVA following Bonferroni's Multiple Comparison Test. It is noteworthy that ELISA results were statistically analyzed by the Effective Dose (ED) method. In this method, a positive reference serum is used to construct a dose-response curve to which the dose-response curve of the test sera is then compared. All animal experiments were approved by Animal Care and Use Committee of the Faculty of Pharmaceutical Sciences of São Paulo University (CEUA/FCF 025.2018-P567).

Results: Following intragastric administration, elevated anti-RV IgG effective doses $50 \%$ were measured in week 5, and these effective doses 50\% were 50-times higher than the negative controls. The suspension of RV administered intraperitoneally was found to induce a higher systemic immune response than the group vaccinated intragastrically with RV loaded CMCS Nps. However, this was not observed for the local antibody levels. Local sIgA antibodies analyzed in saliva 7 days after the third boosting dose were found to be significantly higher with RV loaded CMCS Nps following intragastric administration. All mice vaccinated with RV associated to CMCS nanoparticles produced enough neutralizing antibodies to be protect against rabies.

Conclusion: Therefore, it is concluded that CMCS nanoparticles not only enhances systemic and local immune responses against RV after intragastric immunization, but also protects during actual infections, without any additional mucosal adjuvant.

Keywords: Carboxymethyl chitosan (CMCS) nanoparticles; Rabies virus (RV); Mucosal immunization 